\title{
Swedish National Multicenter Study on Head and Neck Cancer of Unknown Primary: Prognostic Factors and Impact of Treatment on Survival
} \author{
Lena Norberg Spak ${ }^{12}$ Isak Notstam ${ }^{13}$ Eva Hammerlid ${ }^{1,2}$

\footnotetext{
${ }^{1}$ Department of Otorhinolaryngology, University of Gothenburg Sahlgrenska Academy, Goteborg, Sweden

2 Department of Otorhinolaryngology - Head and Neck Surgery, Sahlgrenska University Hospital, Goteborg, Sweden

3 Regional Cancer Center in Western Sweden, Gothenburg, Sweden

${ }^{4}$ Department of Oncology, University of Gothenburg Sahlgrenska Academy, Gothenburg, Sweden

${ }^{5}$ Department of Otorhinolaryngology - Head and Neck Surgery, Karolinska University Hospital, Stockholm, Sweden

${ }^{6}$ Department of Oncology, Karolinska University Hospital, Stockholm, Sweden

7 Department of Oncology, Lund University Hospital, Lund, Skåne, Sweden

${ }^{8}$ Department of Otorhinolaryngology - Head and Neck Surgery, Örebro University Hospital, Örebro, Sweden
}

Lars Axelsson ${ }^{1,20}$ Erik Holmberg ${ }^{3,4}$ Jan Nyman ${ }^{4}$ Anders Högmo ${ }^{5}$ Helena Sjödin 6

Maria Gebre-Medhin ${ }^{7}$ Mathias von Beckerath ${ }^{8}$ Tomas Ekberg ${ }^{9} \quad$ Lisa Farnebo ${ }^{10}$ Charbel Talani ${ }^{11}$
Int Arch Otorhinolaryngol 2021;25(3):e433-e442.

\author{
Address for correspondence Lars Axelsson, MD, PhD, Department of \\ Otorhinolaryngology, University of Gothenburg Sahlgrenska \\ Academy, Gröna Stråket 9, Göteborg, Goteborg 41345, Sweden \\ (e-mail: lars.axelsson1978@gmail.com). \\ ${ }^{9}$ Department of Otorhinolaryngology - Head and Neck Surgery, \\ Uppsala University Hospital, Uppsala, Sweden \\ 10 Department of Otorhinolaryngology - Head and Neck Surgery, \\ Linköping University Hospital, Linkoping, Sweden \\ ${ }^{11}$ Department of Oncology, Linköping University Hospital, \\ Linkoping, Sweden \\ 12 Department of Otorhinolaryngology - Head and Neck Surgery, \\ Norrlands University Hospital, Umea, Sweden \\ ${ }^{13}$ Department of Otorhinolaryngology, County Hospital \\ Sundsvall-Harnosand, Sundsvall, Sweden
}

\section{Abstract \\ Keywords \\ - head and neck cancer \\ - cancer of unknown primary \\ - prognostic factors \\ - human papillomavirus \\ - treatment}

Introduction Head and neck cancer of unknown primary (HNCUP) is a rare condition whose prognostic factors that are significant for survival vary between studies. No randomized treatment study has been performed thus far, and the optimal treatment is not established. Objective The present study aimed to explore various prognostic factors and compare the two main treatments for HNCUP: neck dissection and (chemo) radiation vs primary (chemo) radiation.

Methods A national multicenter study was performed with data from the Swedish Head and Neck Cancer Register (SweHNCR) and from the patients' medical records from 2008 to 2012.

Results Two-hundred and sixty HNCUP patients were included. The tumors were HPVpositive in $80 \%$. The overall 5 -year survival rate of patients treated with curative intent was $71 \%$. Age $(p<0.001)$, performance status $(p=0.036)$, and $N$ stage $(p=0.046)$ were significant factors for overall survival according to the multivariable analysis. Treatment with neck dissection and (chemo) radiation (122 patients) gave an overall 5-year survival of $73 \%$, and treatment with primary (chemo) radiation (87 patients) gave an overall 5-year survival of 71\%, with no significant difference in overall or disease-free survival between the 2 groups.

Conclusions Age, performance status, and $\mathrm{N}$ stage were significant prognostic factors. Treatment with neck dissection and (chemo) radiation and primary (chemo) radiation gave similar survival outcomes. A randomized treatment study that includes quality of life is needed to establish the optimal treatment. received

November 10, 2019

accepted

March 25, 2020

published online

September 30, 2020
DOI https://doi.org/

10.1055/s-0040-1712106. ISSN 1809-9777. (c) 2020. Fundação Otorrinolaringologia. All rights reserved.

This is an open access article published by Thieme under the terms of the Creative Commons Attribution-NonDerivative-NonCommercial-License, permitting copying and reproduction so long as the original work is given appropriate credit. Contents may not be used for commercial purposes, or adapted, remixed, transformed or built upon. (https://creativecommons.org/ licenses/by-nc-nd/4.0/)

Thieme Revinter Publicações Ltda., Rua do Matoso 170, Rio de Janeiro, RJ, CEP 20270-135, Brazil 


\section{Introduction}

Head and neck cancer of unknown primary (HNCUP) is a rare form of head and neck cancer, with an incidence of $\sim 0.47 / 100,000$ people per year. ${ }^{1}$ Head and neck cancer of unknown primary is a diagnosis of exclusion that is used when lymph nodes metastases are present in the neck and no primary tumor can be found despite extensive clinical and radiological examination.

Results from previous retrospective studies on HNCUP vary, and suggested prognostic factors in the literature are: age, gender, performance status, smoking, N-stage, extracapsular extension, and HPV (human papillomavirus) status. $^{2-16}$ Various treatment regimens are used, but the optimal treatment is not established, and no randomized treatment study has been performed thus far. ${ }^{17,18}$

A national register for head and neck cancer in Sweden, the Swedish Head and Neck Cancer Register (SweHNCR), was established in $2008 .{ }^{1}$ Approximately $99 \%$ of all newly-diagnosed Swedish head and neck cancer patients have been reported in the register. The diagnostic work-up for HNCUP is similar among hospitals within Sweden. For curative treatment, one of two treatment regimens are used: either neck dissection combined with (chemo) radiation, or primary (chemo) radiation. ${ }^{19}$

The aim of the present national study was for a large group of HNCUP patients to explore prognostic factors for survival and compare treatment with neck dissection and (chemo) radiation versus (chemo) radiation.

\section{Methods}

\section{Study Design}

A national multicenter study on patients with HNCUP was performed after ethics approval was received from the regional ethics committee, in Gothenburg, Sweden. Patients with HNCUP (ICD-10 code C770) registered in the SweHNCR between January 1,2008, and December 31,2012 were examined. Data from the register and from the patients' medical records were analyzed. Only patients with squamous cell carcinoma (SCC) or undifferentiated carcinoma were included. ${ }^{20,21}$

\section{Management of HNCUP}

\section{Diagnostics}

All patients were examined by a specialist in otolaryngologyhead and neck surgery. A fine-needle aspiration was performed from the neck mass, and combined with a core-needle biopsy or an open biopsy to get a definitive diagnosis. ${ }^{22}$ The diagnostics followed national guidelines. ${ }^{20}$ Human papillomavirus analysis was performed, but it was not routine during the time of the present study and was, therefore, not performed for all the patients. P16 immunostaining was used as the primary determinant of HPV status for 39 patients. Polymerase chain reaction was performed in 35 patients and in situ hybridization in 25 patients. Positron emission tomography/computed tomography (PET/CT) was performed for most patients, but was not routine during the time of this study; alternatively, magnetic resonance imaging (MRI) was performed. A panendoscopy including an examination of the pharynx, larynx, esophagus, and lungs was performed, and bilateral tonsillectomy, and biopsies of the base of the tongue and the nasopharynx were performed. The Union for International Cancer Control (UICC) 7th edition was used for tumor classification.

\section{Treatment}

The patients were discussed at a multidisciplinary conference for tumor staging and treatment recommendations. There are two recommended treatment protocols for HNCUP in the Swedish national guidelines ${ }^{23}: 1$. neck dissection followed by (chemo) radiation or 2. (chemo) radiation. For patients treated with definitive radiotherapy, chemotherapy was used in most university hospitals if no contraindications were present and administered concomitantly. Cisplatin was used as the chemotherapeutic agent.

The neck dissection was either radical neck dissection (neck level 1-5) or modified radical neck dissection (preserving the accessory nerve, internal jugular vein and/or the sternocleidomastoid muscle). Radiation dosage was 68 to 70 Gy administered in $2 \mathrm{~Gy}$ fractions to tumor volumes and 46 to $50 \mathrm{~Gy}$ to adjuvant neck volumes. Intensity-modulated radiotherapy (IMRT) was performed in most cases. Oropharynx was always irradiated and hypopharynx was included in some cases. If retropharyngeal nodes or nodes in level $V$ were present, the nasopharynx was also irradiated. The radiation dose volumes were similar regardless of whether neck dissection was performed or not. In patients receiving postoperative radiation, chemotherapy was administered if high risk features were present. ${ }^{24}$ Salvage surgery was performed in patients treated with (chemo) radiation and evidence of remaining resectable tumor.

\section{Follow-up}

After the treatment was completed, the patients were followed for 5 years with check-ups. A CT or MRI was performed 3 months after the end of the radiation treatment for evaluation of the treatment effect. Imaging was repeated in patients with suspected recurrences.

\section{Statistics}

The results are presented as the mean, standard deviation, median, minimum and maximum for continuous variables, and as numbers and percentages for categorical variables. For comparisons between two groups, the Mann-Whitney U test was used for continuous variables, and the nonparametric test was used for ordered categorical variables. ${ }^{25,26}$ The Fisher exact test was used for nonordered categorical variables and for dichotomous variables. A survival analysis was performed to analyze the time to death and tumor recurrence. KaplanMeier plots were used to describe overall survival and diseasefree survival for the study group and for the subgroups. Comparisons of mortality between subgroups were analyzed using a log-rank test for categorical variables.

Univariable and multivariable Cox proportional hazard regression analyses were used to select independent predictors for overall survival and disease-free survival. All 
significance tests were 2-tailed and were conducted at the $5 \%$ significance level. StataCorp 2017, Stata: Release 15 Statistical Software (StataCorp LLC, College Station, TX, USA) was used for all statistical analyses. ${ }^{27}$

\section{Results}

\section{Patient Selection}

A total of 292 patients were diagnosed with HNCUP in the SweHNCR during the study period. Thirty-two patients were excluded, in most cases because of histopathology other than squamous cell carcinoma or undifferentiated carcinoma (e.g., malignant melanoma, salivary gland cancer), and, in a few cases, because the primary tumor was found. Finally, 260 patients with HNCUP were included in the study: 216 patients were treated with curative intent and 44 were treated with palliative intent ( $\mathbf{- T a b l e ~} \mathbf{1}$ ).

\section{Diagnostic Work-up}

The histopathological diagnosis was established with fineneedle aspiration in $52 \%$, core-needle biopsy in $16 \%$, and open biopsy in 32\%. Positron emission tomography was performed in $67 \%$, MRI in $23 \%$, and CT in $99 \%$ of the patients. Panendoscopy was performed in $95 \%$ of the patients, bilateral tonsillectomy in $48 \%$, ipsilateral tonsillectomy in $29 \%$, and biopsy of the tonsils in $19 \%$, and no sample was obtained from the tonsils in $4 \%$ (these patients had undergone tonsillectomy in childhood) of the patients. Biopsies of the base of the tongue were performed in $83 \%$, biopsies from the nasopharynx in $75 \%$, and biopsies from the hypopharynx in $20 \%$ of the patients.

Table 1 Patient and tumor data for all patients. Patients were divided by treatment intent and by HPV status of the tumor

\begin{tabular}{|c|c|c|c|c|c|c|c|}
\hline & All & Curative & Palliative & $p$ Value & $\mathrm{HPV}+$ & HPV- & $p$ Value \\
\hline Subjects, $n$ & 260 & 216 & 44 & & 81 & 18 & \\
\hline \multicolumn{8}{|l|}{ Age (years) } \\
\hline Mean (SD) & $65.3(13)$ & $62.5(11)$ & $79.0(12)$ & \multirow[t]{2}{*}{0.017} & $60.5(10)$ & $67.9(13)$ & \multirow[t]{2}{*}{0.017} \\
\hline Median (range) & $64(37-95)$ & $62(37-92)$ & $83(54-95)$ & & $61(37-87)$ & $70(47-91)$ & \\
\hline \multicolumn{8}{|l|}{ Gender, $n(\%)$} \\
\hline Male & $191(73)$ & $165(76)$ & $26(59)$ & \multirow[t]{2}{*}{0.024} & $61(75)$ & $12(67)$ & \multirow[t]{2}{*}{0.56} \\
\hline Female & $69(27)$ & $51(24)$ & $18(41)$ & & $20(25)$ & $6(33)$ & \\
\hline \multicolumn{8}{|l|}{ Smoking habits } \\
\hline Never smoker & $19(34)$ & $17(33)$ & $2(40)$ & \multirow[t]{3}{*}{0.79} & $9(35)$ & $1(50)$ & \multirow[t]{3}{*}{0.51} \\
\hline Former smoker ${ }^{a}$ & $23(41)$ & $22(43)$ & $1(20)$ & & $12(46)$ & $1(50)$ & \\
\hline Smoker & $14(25)$ & $12(24)$ & $2(40)$ & & $5(19)$ & 0 & \\
\hline \multicolumn{8}{|l|}{ Performance status ${ }^{b}$} \\
\hline 0 & $188(80)$ & $179(89)$ & $9(26)$ & \multirow[t]{4}{*}{$<0.001$} & $69(95)$ & $14(82)$ & \multirow[t]{4}{*}{0.32} \\
\hline 1 & $23(10)$ & $19(9)$ & $4(12)$ & & $3(4)$ & $2(12)$ & \\
\hline 2 & $8(3)$ & $3(1)$ & $5(15)$ & & 0 & $1(6)$ & \\
\hline $3-4$ & $16(7)$ & 0 & $16(47)$ & & $1(1)$ & 0 & \\
\hline \multicolumn{8}{|l|}{ Tumor histology } \\
\hline SCC & $234(90)$ & $202(94)$ & $32(73)$ & \multirow[t]{2}{*}{$<0.001$} & $80(99)$ & $15(83)$ & \multirow[t]{2}{*}{0.018} \\
\hline Carcinoma N/S & $26(10)$ & $14(6)$ & $12(27)$ & & $1(1)$ & $3(17)$ & \\
\hline \multicolumn{8}{|l|}{ HPV status } \\
\hline Positive & $81(82)$ & $80(84)$ & $1(25)$ & \multirow[t]{2}{*}{0.018} & . & . & \multirow[t]{2}{*}{. } \\
\hline Negative & $18(18)$ & $15(16)$ & $3(75)$ & & . & . & \\
\hline \multicolumn{8}{|l|}{$\mathrm{N}$ stage } \\
\hline N1 & $62(24)$ & $53(25)$ & $9(22)$ & \multirow[t]{3}{*}{0.006} & $24(30)$ & $3(17)$ & \multirow[t]{3}{*}{0.14} \\
\hline N2 & $160(63)$ & $139(65)$ & $21(51)$ & & $51(63)$ & $12(67)$ & \\
\hline N3 & $34(13)$ & $23(11)$ & $11(27)$ & & $6(7)$ & $3(17)$ & \\
\hline \multicolumn{8}{|l|}{ M stage } \\
\hline M0 & 244 (95) & $216(100)$ & $28(68)$ & \multirow[t]{2}{*}{$<0.001$} & 80 (99) & $16(89)$ & \multirow[t]{2}{*}{0.084} \\
\hline M1 & $13(5)$ & 0 & $13(32)$ & & $1(1)$ & $2(11)$ & \\
\hline
\end{tabular}

Abbreviation: HPV, human papillomavirus; SCC, squamous cell carcinoma; SD, standard deviation.

${ }^{\mathrm{a}}$ Former smoker quit smoking at least 1 year ago.

bWHO Performance status. 


\section{Patient and Tumor Characteristics}

The curatively treated patients had a median age of 62 years, $76 \%$ were male, and $90 \%$ had a World Health Organization (WHO) performance status 0 , which can be seen in - Table 1 . Tumor histopathology was SCC in 94\% and undifferentiated carcinoma in $6 \%$ of the cases. The most common $\mathrm{N}$ stage was $\mathrm{N} 2$, which constituted $65 \%$ of the patients, while $25 \%$ were $\mathrm{N} 1$, and $11 \%$ were $\mathrm{N} 3$ tumors.

The palliative treated patients $(n=44)$ were older (median age 83 vs 62 years, $p=0.017$ ), had poorer WHO performance status $(p<0.001)$ and a more advanced $N$ stage $(n=0.006)$ compared with the curatively treated patients (-Table 1). Thirty-two percent of the patients had distant metastases. The median survival for the palliative treated patients was 6.2 months (-Fig. 1A).

\section{Survival and Recurrence}

The overall 2-, 5- and 8-year survival rates for the patients treated with curative intent were $86 \%, 71 \%$, and $68 \%$, respectively ( - Fig. 1A). The 2- and 5-year disease-free survival rates were $83 \%$ and $70 \%$, respectively ( - Fig. 1B). Seventeen patients (8\%) had progressive disease during treatment and never achieved complete remission. Twenty patients $(9 \%)$ experienced a recurrence within 5 years: 5 patients had an emergence of the primary tumor, 4 patients had regional recurrence, 9 patients had distant metastases, and 2 patients had recurrence at 2 or more locations. Of the patients who died within 5 years from diagnosis, $55 \%$ died with the tumor and $45 \%$ without tumor.

\section{Prognostic Factors}

Advanced age was a significant factor for overall survival (hazard ratio 1.076, $p<0.001$, - Table 2). The overall survival differed significantly among different age groups $(p<0.001$, -Fig. 1C), as did disease-free survival $(p<0.001)$. Patients 70 years of age or older had a significantly worse overall 5 -year survival rate than the remaining patients $(p<0.001)$. Patients 60 to 69 years of age had a significantly worse survival than patients younger than 60 years $(p<0.001)$. No significant difference was observed in overall survival between genders ( - Fig. 1D, - Table 2). No significant difference was observed in overall survival among smokers, former smokers, and lifetime never-smokers (- Table 2 ). Survival differed significantly between patients with different WHO performance statuses $(\boldsymbol{p}<0.001$, - Fig. 1E, - Table 2). No significant difference was seen in overall survival between patients with SCC and undifferentiated carcinoma ( - Table 2 ). The overall survival differed significantly for patients with different tumor $\mathrm{N}$ stages $(p=$ 0.002, - Fig. 1F, - Table 2), as patients with N3 tumors had a significantly worse prognosis than the other patients $(p=$ 0.003 ), and patients with $\mathrm{N} 2$ tumors had a significantly worse prognosis than patients with $\mathrm{N} 1$ tumors $(p=0.045$, - Table 2$)$. Human papillomavirus diagnostics was performed in 95 of 216 patients (44\%, - Table 1). The tumors were HPV-positive in $84 \%$ of the cases. The patients with HPV-positive tumors were significantly younger (61 years vs 70 years, $p=0.02$ ) and were more likely to have SCC tumor histology than undifferentiated carcinoma compared with patients with HPV-negative tumors (99\% vs $83 \%, p=0.02)$. Neither the overall survival $(p=$
0.39, - Fig. 1G, - Table 2) nor the disease-free survival ( $p=$ 0.45 ) differed significantly between patients with HPV-positive and those with HPV-negative tumors.

\section{Treatment}

The patients treated with curative intent were divided into three groups based on treatment ( - Table 3 ). Treatment A consisted of neck dissection combined with (chemo) radiation (122 patients, of whom 104 patients underwent neck dissection before (chemo) radiation and 18 patients underwent neck dissection after (chemo) radiation). Treatment B consisted of (chemo) radiation (87 patients), and treatment $C$ consisted of neck dissection ( 7 patients). The patients who had surgery as sole treatment modality either refused or were judged not to withstand postoperative radiotherapy.

The surgery consisted of a radical neck dissection in 12 patients (9\%), a modified radical neck dissection in 92 patients (71\%) and a selective neck dissection (in most cases a supraomohyoid neck dissection) in 25 patients (19\%) ( - Table 3 ). The radiation dose given to the curatively treated patients was 64 to $70 \mathrm{~Gy}$ in 163 patients (80\%), the dose per fraction was $2 \mathrm{~Gy}$ in 189 patients (94\%), 51\% of the patients were irradiated to the oropharynx and $65 \%$ were irradiated bilaterally to the neck ( - Table 3 ). Chemotherapy was given to 71 patients (35\%); $79 \%$ of patients were given concomitant chemotherapy. In most cases, concomitant chemotherapy consisted of weekly cisplatin. Induction chemotherapy was a combination of cisplatin and 5-fluorouracil.

An aim of this study was to compare treatment outcome between combined modality treatment (treatment $A$ ) and definitive (chemo) radiation (treatment $B$ ). The patient groups did not differ regarding age, smoking habits, WHO performance status, tumor histology, HPV status, and N stage ( - Table 3 ). The treatment $\mathrm{A}$ group contained significantly more male patients than the treatment $B$ group ( $81 \%$ vs $68 \%, p=0.03$ ). Patients who received treatment $B$ had a significantly higher radiation dose (93\% vs 70\% 64-70 Gy, $p<0.001$ ), were more often irradiated to the bilateral neck ( $75 \%$ vs $58 \%, p=0.02$ ), and were more prone to receive chemotherapy ( $53 \%$ vs $25 \%, p<.001$ ).

No significant differences were observed in overall or disease-free survival between patients treated with treatment A and those treated with treatment B ( $\mathbf{- F i g . ~} \mathbf{1 H}$, -Table 2). The overall 5-year survival of patients treated with treatment A was $73 \%$, and that of patients treated with treatment B was $71 \%$. The small group of patients treated with treatment $C$ exhibited a significantly worse overall survival than patients in the treatment $A$ and $B$ groups (hazard ratio 3.07 C versus A, $p=0.019$, - Table 2, - Fig. 1H).

\section{Multivariable Analysis}

Four factors were significant for overall survival in the univariable analysis in patients who were treated with curative intent: age, performance status, $\mathrm{N}$ stage and treatment (-Table 2). These four factors were included in the multivariable analysis of overall survival. Age (hazard ratio 1.072 per year, $p<0.001$ ), performance status (hazard ratio 2.12 WHO 1 versus $0, p=0.036$ ), and $\mathrm{N}$ stage (hazard ratio $2.63 \mathrm{~N} 3$ versus $\mathrm{N} 1, p=0.046$ ) were significant factors in the 

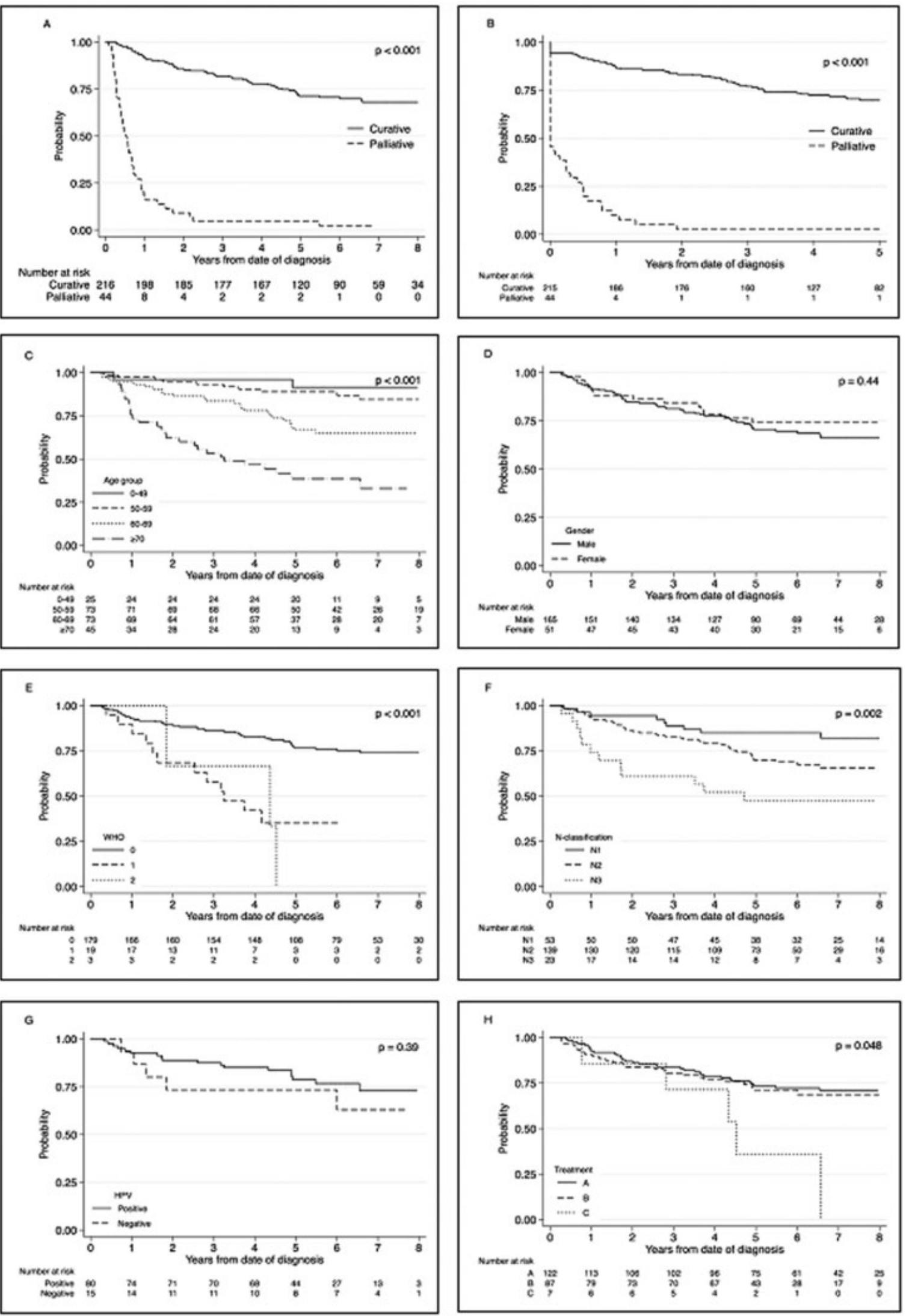

Fig. 1 (A-H) Kaplan-Meier plots of overall survival probability (A, C, D, E, F, G and H) and disease-free survival (B), for various prognostic factors. In $\mathrm{C}-\mathrm{H}$, only patients with curative treatment were included. The number of patients at risk is shown at the bottom of the figures. Significance levels were calculated with a log-rank test. A, B Treatment intent. C Age groups. D Gender. E Performance status (WHO). F N classification. G HPV status. $\mathrm{H}$ Treatment. Treatment $\mathrm{A}=$ neck dissection and (chemo)radiation, treatment $\mathrm{B}=$ (chemo)radiation, and treatment $\mathrm{C}=$ neck dissection. 
Table 2 Uni- and multivariable Cox regression analyses on overall survival for patients treated with curative intent

\begin{tabular}{|c|c|c|c|c|c|c|}
\hline & \multicolumn{3}{|c|}{ Univariable analyses } & \multicolumn{3}{|c|}{ Multivariable analysis } \\
\hline & $n$ & $\mathrm{HR}(95 \% \mathrm{Cl})$ & $p$ & $\mathrm{n}$ & $\mathrm{HR}(95 \% \mathrm{Cl})$ & $p$ \\
\hline \multicolumn{7}{|l|}{ Age (years) } \\
\hline Continuous & 216 & $1.076(1.053-1.099)$ & $<0.001$ & 201 & $1.072(1.042-1.102)$ & $<0.001$ \\
\hline \multicolumn{7}{|l|}{ Gender } \\
\hline Male & 165 & 1 & & & . & \\
\hline Female & 51 & $0.79(0.43-1.44)$ & 0.44 & . & . & . \\
\hline \multicolumn{7}{|l|}{ Smoking habits } \\
\hline Never smoker & 17 & 1 & & & $\cdot$ & \\
\hline Former smoker ${ }^{\mathrm{a}}$ & 22 & $0.73(0.18-2.94)$ & 0.66 & & . & . \\
\hline Smoker & 12 & $1.01(0.23-4.53)$ & 0.99 & . & . & . \\
\hline \multicolumn{7}{|l|}{ Performance status ${ }^{\mathrm{b}}$} \\
\hline 0 & 179 & 1 & & 179 & 1 & \\
\hline 1 & 19 & $3.90(2.04-7.47)$ & $<0.001$ & 19 & $2.12(1.05-4.26)$ & 0.036 \\
\hline 2 & 3 & $5.58(1.72-18.1)$ & 0.004 & 3 & $2.92(0.80-10.7)$ & 0.10 \\
\hline \multicolumn{7}{|l|}{ Tumor histology } \\
\hline SCC & 202 & 1 & & . & . & . \\
\hline Carcinoma N/S & 14 & $1.54(0.66-3.58)$ & 0.31 & & . & 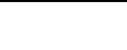 \\
\hline \multicolumn{7}{|l|}{ HPV status } \\
\hline Positive & 80 & 1 & 0.39 & . & . & . \\
\hline Negative & 15 & $1.54(0.57-4.16)$ & & . & . & . \\
\hline \multicolumn{7}{|l|}{$N$ stage } \\
\hline N1 & 53 & 1 & & 51 & 1 & \\
\hline N2 & 139 & $2.08(1.01-4.27)$ & 0.045 & 130 & $1.82(0.88-3.81)$ & 0.11 \\
\hline N3 & 23 & $4.39(1.85-10.4)$ & 0.001 & 20 & $2.63(1.02-6.78)$ & 0.046 \\
\hline \multicolumn{7}{|l|}{ Treatment } \\
\hline A & 122 & 1 & & 110 & 1 & \\
\hline $\mathrm{B}$ & 87 & $1.11(0.66-1.86)$ & 0.70 & 84 & $1.44(0.83-2.50)$ & 0.20 \\
\hline $\mathrm{C}$ & 7 & $3.07(1.20-7.87)$ & 0.019 & 7 & $0.63(0.20-1.97)$ & 0.42 \\
\hline
\end{tabular}

Abbreviations: $\mathrm{Cl}$, confidence interval; HR, hazard ratio; SCC, squamous cell carcinoma.

Treatment A, neck dissection in combination with (chemo)radiation. Treatment B, (chemo)radiation. Treatment C, neck dissection.

${ }^{a}$ Former smoker quit smoking at least 1 year ago.

bWHO Performance status.

multivariable analysis. Treatment was not a significant factor for overall survival in the multivariable analysis.

Human papillomavirus status was available in 95 of the patients (44\%). Since HPV is an interesting prognostic factor for HNCUP, a second multivariable analysis of the curative patients was performed. Only age (hazard ratio $1.09, p=$ 0.003 ) was a significant independent factor for overall survival, while the other factors, including HPV status (hazard ratio $1.25, p=0.72$ ), were not significant for overall survival in this multivariable analysis.

\section{Discussion}

The SweHNCR covers 99\% of all head and neck cancer patients in Sweden compared with the Swedish Cancer Register, and the current study, therefore, includes close to all patients with
HNCUP in Sweden during the study period. The patients in the study had a thorough medical work-up. Positron emission tomography/computed tomography was performed in $67 \%$ of the patients, which is a high number compared with previous studies (PET was performed in $1 \%$ in a study by Grau et al, ${ }^{2} 9 \%$ in a study by Wallace et $\mathrm{al}^{20}$ and $50 \%$ in a study by Frank et $\mathrm{al}^{21}$ ). As in previous studies, the majority of our patients were male, ${ }^{28,29}$ and the majority of the tumors were SCC with some cases of undifferentiated carcinoma. ${ }^{8,20}$

The overall 5-year survival rate for patients treated with curative intent was $71 \%$, which is a similar outcome as for the total population of head and neck cancer patients in Sweden. ${ }^{1}$ This is similar to newer studies of HNCUP, $, 7,21$ but better than the survival rate reported in older studies. ${ }^{9,30}$ Seventeen percent of the patients had either progressive disease or tumor recurrence within 5 years of the current 
Table 3 Patient, tumor and treatment data for patients treated with curative intent, divided by treatment

\begin{tabular}{|c|c|c|c|c|}
\hline & Treatment A & Treatment B & $p A$ vs $B$ & Treatment C \\
\hline Subjects, $n$ & 122 & 87 & & 7 \\
\hline \multicolumn{5}{|l|}{ Age (years) } \\
\hline Mean (SD) & $62.1(11)$ & $61.6(10)$ & & $82.0(8)$ \\
\hline Median (range) & $61(37-89)$ & $60(39-92)$ & 0.83 & $84(66-89)$ \\
\hline \multicolumn{5}{|l|}{ Gender, $n(\%)$} \\
\hline Male & $99(81)$ & $59(68)$ & & $7(100)$ \\
\hline Female & $23(19)$ & $28(32)$ & 0.034 & 0 \\
\hline \multicolumn{5}{|l|}{ Smoking habits } \\
\hline Never smoker & $10(31)$ & $6(33)$ & & 1 \\
\hline Former smoker ${ }^{\mathrm{a}}$ & $14(44)$ & $8(44)$ & & 0 \\
\hline Smoker & $8(25)$ & $4(22)$ & 0.83 & 0 \\
\hline \multicolumn{5}{|l|}{ Performance status ${ }^{\mathrm{b}}$} \\
\hline 0 & $101(92)$ & $74(88)$ & & $4(57)$ \\
\hline 1 & $8(7)$ & $9(11)$ & & $2(29)$ \\
\hline 2 & $1(1)$ & $1(1)$ & 0.42 & $1(14)$ \\
\hline \multicolumn{5}{|l|}{ Tumor histology } \\
\hline SCC & $114(93)$ & $82(94)$ & & $6(86)$ \\
\hline Carcinoma N/S & $8(7)$ & $5(6)$ & 0.55 & $1(14)$ \\
\hline \multicolumn{5}{|l|}{ HPV status } \\
\hline Positive & $55(85)$ & $24(86)$ & & $1(50)$ \\
\hline Negative & $10(15)$ & $4(14)$ & 1.0 & $1(50)$ \\
\hline \multicolumn{5}{|l|}{$\mathrm{N}$ stage } \\
\hline N1 & $27(22)$ & $24(28)$ & & $2(29)$ \\
\hline N2 & $80(66)$ & $55(63)$ & & $4(57)$ \\
\hline N3 & $14(12)$ & $8(9)$ & 0.35 & $1(14)$ \\
\hline \multicolumn{5}{|l|}{ Surgery } \\
\hline RND & $12(10)$ & . & & 0 \\
\hline MRND & $85(70)$ & . & & $7(100)$ \\
\hline SND & $25(20)$ & . & & 0 \\
\hline \multicolumn{5}{|l|}{ Radiation dose } \\
\hline 64-70 Gy & $85(70)$ & $78(93)$ & & . \\
\hline $60 \mathrm{~Gy}$ & $31(26)$ & $6(7)$ & & . \\
\hline$\leq 56 \mathrm{~Gy}$ & $5(4)$ & 0 & $<0.001$ & . \\
\hline \multicolumn{5}{|c|}{ Radiation to the pharynx } \\
\hline All of pharynx & $3(3)$ & $3(4)$ & & . \\
\hline Hypo-oropharynx & $49(41)$ & $27(34)$ & & . \\
\hline Oropharynx & $56(47)$ & $45(56)$ & & . \\
\hline No radiation & $11(9)$ & $5(6)$ & 0.51 & . \\
\hline \multicolumn{5}{|l|}{ Radiation to the neck } \\
\hline Bilateral & $70(58)$ & $62(75)$ & & . \\
\hline Ipsilateral & $51(42)$ & $21(25)$ & 0.017 & . \\
\hline
\end{tabular}


Table 3 (Continued)

\begin{tabular}{|l|l|l|l|l|}
\hline & Treatment A & Treatment B & p A vs B & Treatment C \\
\hline Chemotherapy & & & & \\
\hline Yes & $30(25)$ & $41(53)$ & &. \\
\hline No & $91(75)$ & $37(47)$ & $<0.001$ &. \\
\hline Chemotherapy & & & & \\
\hline Induction & $4(13)$ & $11(27)$ & &. \\
\hline Concomitant & $26(87)$ & $30(73)$ & 0.24 &. \\
\hline
\end{tabular}

Abbreviations: MRND, modified radical neck dissection; RND, radical neck dissection; SCC, squamous cell carcinoma; SND, selective neck dissection, in most cases supraomohyoidal neck dissection.

Treatment A, neck dissection in combination with (chemo)radiation. Treatment B, (chemo)radiation. Treatment C, neck dissection.

${ }^{a}$ Former smoker quit smoking at least 1 year ago.

bWHO Performance status.

study. Previous studies have shown varying recurrence rates; two studies from around the year 2000 reported that more than $50 \%$ of the patients had tumor recurrence, ${ }^{2,9}$ while the results of two more recent studies were similar to the result of the current study, which found the tumor recurrence rate to be $\sim 10 \%{ }^{6,21}$ One can speculate that the large difference in recurrence rates is due to differences in both patient selection, tumor characteristics and in treatment differences.

The patients treated with palliative intent had a poor prognosis: $82 \%$ of the patients were deceased within 1 year, which is comparable with the result of a previous study in which 6 palliative patients had a median survival of 8.5 months. $^{3}$

Advanced age was a significant negative factor for survival, as shown in previous studies. ${ }^{2,3,6}$ The median age of the patients treated with curative intent was 62 years, which is higher than that reported in previous studies. ${ }^{4,6}$ It was noticed that patients older than 70 years died more often during the first year after diagnosis compared with the rest of the patients ( $\mathbf{- F i g . 1 C}$ ). The explanation for this may be that the older patients were more fragile, had more comorbidities and more often died due to treatment side effects. More efforts should be made during the diagnostic work-up to better diagnose any comorbidity before treatment recommendation. Treatment guidelines should take age and comorbidities into greater consideration.

In the study, the patients who were treated with curative intent had a good performance status: $89 \%$ were asymptomatic (WHO 0), which is high compared with previous studies. $^{2,31}$ Patients with WHO 0 had a significantly better survival than patients with WHO 1. This emphasizes the value of performance status as a clinical factor per se, since patients with WHO 0 and 1 were given the same treatment according to the guidelines.

The most common N stage was N2 (63\%), whereas $13 \%$ of the patients were N3, and $24 \%$ were N1, which is similar to what was reported in two recent studies., ${ }^{6,32}$ The overall survival was worse with higher $\mathrm{N}$ stage. This was also seen in some previous studies, 2,8 but in contrast to the current study, other studies showed a similar prognosis between N1 and $\mathrm{N} 2{ }^{3,6}$
Most HNCUP patients were HPV-positive, as seen in previous studies. ${ }^{6,12,32}$ This adds to the evidence that HNCUP is an undiscovered oropharyngeal cancer. The overall and disease-free survival for curatively treated patients with HPV-positive and HPV-negative HNCUP did not differ significantly, and this is in contrast to previous studies, which have shown a significantly better survival rate for HPV-positive patients. $6,11,12$

An aim of this study was to compare the outcomes after treatment with neck dissection combined with (chemo) radiation versus definitive (chemo) radiation. The two treatment groups had similar overall and disease-free survival rates. The patients in the two groups were comparable with respect to background data, but the patients receiving definitive (chemo) radiation (treatment $B$ ) had a significantly higher radiation dose, more bilateral neck radiation and chemotherapy than the patients receiving combination treatment $A$; therefore, the results must be interpreted with caution. The question remains as to whether neck dissection or chemotherapy is needed routinely as part of HNCUP treatment. Most previous studies that compare neck dissection and (chemo) radiation with definitive (chemo) radiation reported results similar to those of the current study, with no statistically significant difference in survival between the two treatments. $6,13,33$ However, some studies found significantly better outcomes for neck dissection combined with (chemo) radiation than for (chemo) radiation only. ${ }^{14,15}$ Another question is whether the most advanced HNCUP (N3 tumors) could benefit from an aggressive treatment regimen that includes both neck dissection and radiation. Unfortunately, a subgroup analysis of only $\mathrm{N} 3$ patients included too few patients for any conclusions to be drawn (14 patients with combined treatment and 8 patients with definitive (chemo) radiation; 5-year survival $57 \%$ vs 33\%, $p=0.28$ ).

A factor that favors neck dissection as part of HNCUP treatment is that a better histopathological diagnosis is achieved than when it is based on fine-needle aspiration only. We have encountered some cases that were first classified as SCC or undifferentiated carcinoma on fine-needle aspiration; those patients then underwent neck dissection, and postoperative analysis showed salivary gland cancer and 
even a case of Warthin tumor. Information of extracapsular extension and perineural growth is achieved with neck dissection, which are important prognostic factors.

The current study was one of the largest studies of HNCUP that compares curative treatment with neck dissection and radiation vs definitive (chemo) radiation with modern treatment regimens (2008-2012). However, a couple of limitations of the study were the nonrandomized design, and that there were differences in the oncologic treatment between the treatment groups. A positive aspect was that the patients in treatment A were treated at some university hospitals, while those with treatment B at some other university hospitals, making difference in patients and tumor factors between the two groups theoretically less likely. Another limitation of the study was the relatively high proportion of missing data for some factors (HPV status and smoking history, both became obligatory variables in the registry after 2013) and lack of some data (resection margin status, extracapsular tumor extension). A final limitation of the study was that information about toxicity and patients' quality of life is missing. If survival is comparable, it is necessary to consider the long-term quality of life and treatment side effects for future treatment guidelines. We recommend a future international randomized study of HNCUP treatment that includes a quality of life measurement to enable the best treatment recommendation that takes both survival and quality of life into consideration.

\section{Conclusion}

Human papillomavirus-positive tumors were common ( $~ 80 \%$ of patients). Age, performance status, and $\mathrm{N}$ stage were significant factors for overall survival in the univariable and multivariable analyses in HNCUP patients treated with curative intent. Treatment with neck dissection combined with (chemo) radiation and primary (chemo) radiation resulted in similar overall and disease-free survival rates. A future randomized treatment study including quality of life measurements is needed to establish treatment recommendations that lead to the most favorable survival and that have the least impact on quality of life.

Funding

Stiftelsen Acta Oto-Laryngologica; The ALF project funding for clinically oriented medical research projects; The Health and Medical Care Committee of the Regional Executive Board, Region Västra Götaland.

\section{Conflict of Interest}

Dr. Axelsson reports grants from The Foundation ACTA Oto-Laryngologica, The Health and Medical Care Committee of the Regional Executive Board, Region VästraGötaland, and from The ALF project funding for clinically oriented medical research projects, during the conduct of the study.

Dr. Hammerlid reports grants from The Health and Medical Care Committee of the Regional Executive Board, Region VästraGötaland, and from The ALF project funding for clinically oriented medical research projects, during the conduct of the study.

\section{References}

1 Swedish Head and Neck Cancer Register (SweHNCR). http://www. cancercentrum.se/samverkan/cancerdiagnoser/huvudoch-hals/kvalitetsregister

2 Grau C, Johansen LV, Jakobsen J, Geertsen P, Andersen E, Jensen BB. Cervical lymph node metastases from unknown primary tumours. Results from a national survey by the Danish Society for Head and Neck Oncology. Radiother Oncol 2000;55(02):121-129

3 Huang CC, Tseng FY, Yeh TH, et al. Prognostic factors of unknown primary head and neck squamous cell carcinoma. Otolaryngol Head Neck Surg 2008;139(03):429-435

4 Beldì D, Jereczek-Fossa BA, D'Onofrio A, et al. Role of radiotherapy in the treatment of cervical lymph node metastases from an unknown primary site: retrospective analysis of 113 patients. Int J Radiat Oncol Biol Phys 2007;69(04):1051-1058

5 Boscolo-Rizzo P, Gava A, Da Mosto MC. Carcinoma metastatic to cervical lymph nodes from an occult primary tumor: the outcome after combined-modality therapy. Ann Surg Oncol 2007;14(05): 1575-1582

6 Axelsson L, Nyman J, Haugen-Cange H, et al. Prognostic factors for head and neck cancer of unknown primary including the impact of human papilloma virus infection. J Otolaryngol Head Neck Surg 2017;46(01):45

7 Zhou MJ, van Zante A, Lazar AA, et al. Squamous cell carcinoma of unknown primary of the head and neck: Favorable prognostic factors comparable to those in oropharyngeal cancer. Head Neck 2018;40(05):904-916

8 Erkal HS, Mendenhall WM, Amdur RJ, Villaret DB, Stringer SP. Squamous cell carcinomas metastatic to cervical lymph nodes from an unknown head-and-neck mucosal site treated with radiation therapy alone or in combination with neck dissection. Int J Radiat Oncol Biol Phys 2001;50(01):55-63

9 Iganej S, Kagan R, Anderson P, et al. Metastatic squamous cell carcinoma of the neck from an unknown primary: management options and patterns of relapse. Head Neck 2002;24(03): 236-246

10 Strojan P, Anicin A. Combined surgery and postoperative radiotherapy for cervical lymph node metastases from an unknown primary tumour. Radiother Oncol 1998;49(01):33-40

11 Sivars L, Näsman A, Tertipis N, et al. Human papillomavirus and p53 expression in cancer of unknown primary in the head and neck region in relation to clinical outcome. Cancer Med 2014;3 (02):376-384

12 Keller LM, Galloway TJ, Holdbrook T, et al. p16 status, pathologic and clinical characteristics, biomolecular signature, and longterm outcomes in head and neck squamous cell carcinomas of unknown primary. Head Neck 2014;36(12):1677-1684

13 Aslani M, Sultanem K, Voung T, Hier M, Niazi T, Shenouda G. Metastatic carcinoma to the cervical nodes from an unknown head and neck primary site: Is there a need for neck dissection? Head Neck 2007;29(06):585-590

14 Hauswald H, Lindel K, Rochet N, Debus J, Harms W. Surgery with complete resection improves survival in radiooncologically treated patients with cervical lymph node metastases from cancer of unknown primary. Strahlenther Onkol 2008;184(03): 150-156

15 Bataini JP, Rodriguez J, Jaulerry C, Brugere J, Ghossein NA. Treatment of metastatic neck nodes secondary to an occult epidermoid carcinoma of the head and neck. Laryngoscope 1987;97(09): $1080-1084$

16 Coster JR, Foote RL, Olsen KD, Jack SM, Schaid DJ, DeSanto LW. Cervical nodal metastasis of squamous cell carcinoma of unknown origin: indications for withholding radiation therapy. Int J Radiat Oncol Biol Phys 1992;23(04):743-749 
17 Strojan P, Ferlito A, Langendijk JA, et al. Contemporary management of lymph node metastases from an unknown primary to the neck: II. a review of therapeutic options. Head Neck 2013;35(02): 286-293

18 Eskander A, Ghanem T, Agrawal A; Education Committee of American Head and Neck Society (AHNS). AHNS Series: Do you know your guidelines? Guideline recommendations for head and neck cancer of unknown primary site. Head Neck 2018;40(03): 614-621

19 Farnebo L, Laurell G, Mäkitie A. A Nordic survey on the management of head and neck CUP. Acta Otolaryngol 2016;136(11): 1159-1163

20 Wallace A, Richards GM, Harari PM, et al. Head and neck squamous cell carcinoma from an unknown primary site. Am J Otolaryngol 2011;32(04):286-290

21 Frank SJ, Rosenthal DI, Petsuksiri J, et al. Intensity-modulated radiotherapy for cervical node squamous cell carcinoma metastases from unknown head-and-neck primary site: M. D. Anderson Cancer Center outcomes and patterns of failure. Int J Radiat Oncol Biol Phys 2010;78(04):1005-1010

22 Strojan P, Ferlito A, Medina JE, et al. Contemporary management of lymph node metastases from an unknown primary to the neck: I. A review of diagnostic approaches. Head Neck 2013;35(01): 123-132

23 Swedish national care program for head and neck cancer. https:// www.cancercentrum.se/samverkan/cancerdiagnoser/huvudoch-hals/vardprogram/

24 Pignon JP, le Maître A, Maillard E, Bourhis J; MACH-NC Collaborative Group. Meta-analysis of chemotherapy in head and neck cancer (MACH-NC): an update on 93 randomised trials and 17,346 patients. Radiother Oncol 2009;92(01):4-14
25 Cuzick J. A method for analysing case-control studies with ordinal exposure variables. Biometrics 1985;41(03):609-621

26 KA Stepniewska DA. snp4: Non-parametric test for trend across ordered groups. Stata Technical Bulletin,1992Reprints, vol 2. 169College Station, TX: Stata Press

27 Reference: StataCorp. Stata: Release 15. Statistical Software. College Station, TX: StataCorp LLC; 2017

28 Fakhrian K, Thamm R, Knapp S, et al. Radio(chemo)therapy in the management of squamous cell carcinoma of cervical lymph nodes from an unknown primary site. A retrospective analysis. Strahlenther Onkol 2012;188(01):56-61

29 Ligey A, Gentil J, Créhange G, et al. Impact of target volumes and radiation technique on loco-regional control and survival for patients with unilateral cervical lymph node metastases from an unknown primary. Radiother Oncol 2009;93(03):483-487

30 Fernández JA, Suárez C, Martínez JA, Llorente JL, Rodrigo JP, Alvarez JC. Metastatic squamous cell carcinoma in cervical lymph nodes from an unknown primary tumour: prognostic factors. Clin Otolaryngol Allied Sci 1998;23(02):158-163

31 Klem ML, Mechalakos JG, Wolden SL, et al. Intensity-modulated radiotherapy for head and neck cancer of unknown primary: toxicity and preliminary efficacy. Int J Radiat Oncol Biol Phys 2008;70(04):1100-1107

32 Motz K, Qualliotine JR, Rettig E, Richmon JD, Eisele DW, Fakhry C. Changes in Unknown Primary Squamous Cell Carcinoma of the Head and Neck at Initial Presentation in the Era of Human Papillomavirus. JAMA Otolaryngol Head Neck Surg 2016;142 (03):223-228

33 Balaker AE, Abemayor E, Elashoff D, St John MA. Cancer of unknown primary: does treatment modality make a difference? Laryngoscope 2012;122(06):1279-1282 